\title{
Quasielastic Light Scattering Measurements of Polystyrene Latices and Conformation of Poly(oxyethylene) Adsorbed on the Latices
}

\author{
Tadaya KATo, Kimiaki NaKamura, Masami KaWAGUCHI, \\ and Akira TAKAHASHI \\ Department of Industrial Chemistry, Faculty of Engineering, \\ Mie University, Tsu, Mie 514, Japan.
}

(Received May 18, 1981)

\begin{abstract}
One of the most useful methods for particle size determination of polymer dispersions, the quasielastic light scattering technique (QELS), was applied to determine the thickness of the layers of monodisperse poly(oxyethylene) adsorbed on the polystyrene latex surface. The molecular weights ranged from $2 \times 10^{4}$ to $130 \times 10^{4} \mathrm{~g} \mathrm{~mol}^{-1}$. On the basis of the experimental results for both adsorbance and thickness of an adsorbed polymer layer; the conformation of the adsorbed polymer is discussed. The hydrodynamic thickness of the adsorbed polymer layer is nearly equal to the dimensions of an isolated polymer in bulk solution. Adsorbance data indicate that the adsorbed polymers must considerably overlap one another or are laterally compressed. The molecular weight dependence of the adsorbed layer thickness is related to the excluded-volume effect of the polymer chain in bulk solution.

KEY WORDS Quasielastic Light Scattering / Particle Size Determination / Thickness of Adsorbed Polymer Layer / Polystyrene Latex / Monodisperse Poly(oxyethylene) / Adsorbance / Conformation of Adsorbed Polymer / Diffusion Coefficient / Stokes Radius /
\end{abstract}

It is well recognized that analysis of the conformation of a polymer adsorbed on a surface is very important for understanding polymer adsorption behavior. In the last two decades, several theories $^{1-7}$ concerning the polymer conformation at interfaces have been developed and are based on several conformational models for adsorbed linear polymers, such as the loop-train-tail conformation and/or the loop-train conformation. However, no theory has yet adequately explained the experimental data completely. In order to compare experimental results with theory, many quantities must be known, e.g., the surface density of active sites for segment adsorption, the fraction of the covered surface, and the adsorbance and thickness of the adsorbed layer. Among these parameters, the adsorbance and thickness of the adsorbed layer should be measured first. Ellipsometry has been used for measuring these two quantities for flat surfaces. ${ }^{8,9}$ We have reported experimental results derived by ellipsometry elsewhere. ${ }^{10-12}$ For the study of polymer adsorption onto the surface of polymer disper- sions, the quasielastic light scattering technique (QELS) is recommended by Garvey et al. ${ }^{13}$ and by Morrissey and Han. ${ }^{14}$ The adsorption study on polymer dispersions is very important for elucidating the colloid stability in dispersions.

In the present paper, we first describe QELS measurements of the radii of monodisperse polystyrene latices for particles ranging in diameter from 0.1 to $1.0 \mu \mathrm{m}$. These measurements were made so as to confirm that the QELS method was the most precise and convenient way for determining particle size. Next, we report the thicknesses of adsorbed layers of monodisperse poly(oxyethylene) on a polystyrene latex surface measured over a wide range of molecular weight from $2 \times 10^{4}$ to $130 \times 10^{4}$ $\mathrm{g} \mathrm{mol}^{-1}$. In the work of Garvey, Tadros and Vincent, ${ }^{15}$ somewhat lower molecular weight fractions of poly(vinyl alcohol) were used. The thicknesses were calculated from the Stokes radii of polystyrene latex particles with an adsorbed polymer layer and estimated from the translational diffusion coefficients determined by QELS. More- 
over, measurement of adsorbance was performed by centrifugation followed by gel-permeation chromatography. Finally, conformation of the adsorbed poly(oxyethylene) molecule on the polystyrene latex surface is discussed in terms of experiment and theory ${ }^{16-18}$ comparison.

\section{EXPERIMENTAL}

\section{Materials}

All chemicals used were of reagent grade; the polystyrene monomer and organic solvents were used after distillation. Water was doubly distilled by Pyrex distillation apparatus.

The poly(oxyethylene) (abbreviated PEO) were standard samples for aqueous gel permeation chromatography (GPC) and purchased from Toyo Soda MFG Co. These samples were very narrow in molecular weight distribution. Their molecular characterization was evaluated by viscosity, light scattering, and GPC on aqueous solutions.

Intrinsic viscosities $[\eta]$ were measured in water at $25^{\circ} \mathrm{C}$ using a conventional capillary viscometer of the Ubbelohde suspended-level type. The weightaverage molecular weights $M_{w}$ were measured by light scattering. The molecular weight distributions of the polymer samples were measured by aqueous GPC with four TSK PW-type 2 feet columns. The chromatographic solvents were water and $0.08 \mathrm{M}$ Tris- $\mathrm{HCl}$ buffer $(\mathrm{pH}=7.9)$.

\section{Polystyrene Latices}

Dow uniform polystyrene latex particles (DPSL) purchased from the Dow Chemical Co. were used as monodisperse spherical substrates of $109 \mathrm{~nm}$ diameter. A DPSL solution of $10 \%$ solid was diluted with distilled water to $10^{-2} \mathrm{~g} \mathrm{ml}^{-1}$ concentration, and the diluted solution was dialyzed for one month in order to remove surfactants. The other latices (series PSL) were prepared by emulsion polymerization under a nitrogen gas atmosphere using potassium persulfate as the initiator without an emulsifier. ${ }^{19}$ Solutions of these latices were also extensively dialyzed beofore used. Measurement of the mean particle diameter and particle size distribution was made with a transmission electron microscope (EM).

\section{Quasielastic Light Scattering Measurements}

It is important, in practice, to obtain the time dependent photocount autocorrelation function $G_{n}(\tau)$ of the light scattered from polymer dispersions. $G_{n}(\tau)$ is given by,

$$
G_{n}(\tau)=A\left\{1+\beta\left|g^{(1)}(\tau)\right|^{2}\right\}
$$

where $\tau$ is the correlation delay time, $A$ is the background at a long delay time and $\beta$ is an adjustable parameter. $g^{(1)}(\tau)$ is the scattered electric field correlation function. For monodisperse and spherically symmetrical particles, $g^{(1)}(\tau)$ has the form,

$$
\left|g^{(1)}(\tau)\right|=\exp \left(-\Gamma_{\mathrm{D}} \tau\right)
$$

where $\Gamma_{\mathrm{D}}=D q^{2}$ with $D$ the translational diffusion coefficient, $q=(4 \pi / \lambda) \sin (\theta / 2)$ the magnitude of the scattering vector, $\theta$ the scattering angle, and $\lambda$, the wavelength in the continuous medium.

By the Stokes-Einstein relation,

$$
D=\frac{k T}{6 \pi \eta_{\circ} R_{\mathrm{h}}}
$$

the Stokes radius $R_{\mathrm{h}}$ can be calculated, where $k$ is the Boltzmann constant, $T$ is the absolute temperature, and $\eta_{0}$ is the viscosity of the medium. The thickenss $t_{\mathrm{h}}$ of the adsorbed polymer layer may be estimated from the difference between the Stokes radius of the bare particle and that of the particle with the adsorbed polymer layer.

Light scattering measurements were made with an Union Giken LS 601 A automatic light scattering analyser. Vertically polarized light of $488 \mathrm{~nm}$ wavelength from an Ar ion laser of NEC GLG3000 was used as the light source. A cylindrical cell of $10 \mathrm{~mm}$ inner diameter was placed at the center of the goniometer in a thermostated bath filled with toluene. The stock solution of DPSL was purified by dialysis and then diluted by PEO solutions of known concentrations. The PEO-latex mixed solutions thus prepared were immediately clarified by filtration through a Millipore filter of pore size $1.2 \mu \mathrm{m}$, and directly poured into the scattering cell. This time was taken as the zero adsorption time. The light scattering intensity of PEO in the mixed solution was negligible in comparison with that of latex particles. The process of adsorption of PEO onto DPSL particles was followed by QELS measurements at appropriate time intervals. Usually, one QELS measurement took five minutes, thus, the measured $G_{n}{ }^{\prime}(\tau)$ was assumed to be the average value over this time interval. The scattering 
angles were varied from 10 to $150^{\circ}$. The intensity fluctuation of the incident laser light was corrected by monitoring the intensity of light reflected from a half mirror with a silicon photodiode at right angles to the incident beam. The output of the photocounting pulses from the photomultiplier was amplified, discriminated, and fed into a microcomputer of SORD M200 (SORD Computer Systems, Inc.) by an Union Giken Interface. The photocount autocorrelation function was calculated by the timeinterval data processing method. ${ }^{20}$ The computernormalized photocount autocorrelation function $G_{n}{ }^{\prime}(\tau)$, defined by $G_{n}{ }^{\prime}(\tau)=G_{n}(\tau) / A$, was printed out on an $\mathrm{X}-\mathrm{Y}$ recorder as a function of the delay time $\tau$. The $\Gamma_{\mathrm{D}}$ values in eq 2 were estimated by the curve fitting method with a single exponential function and/or by the cumulant data analysis. ${ }^{21,22}$

The adsorbances of PEO on DPSL were measured as follows. A solution for adsorbance measurement was made by mixing $2 \mathrm{ml}$ of DPSL solution of $7.128 \times 10^{-3} \mathrm{~g} \mathrm{ml}^{-1}$ with $10 \mathrm{ml}$ of PEO solution of known concentration. The solution was stored in an incubator at $25^{\circ} \mathrm{C}$ for 2 days until the adsorption equilibrium was reached. The solution was then centrifuged at $15,000 \mathrm{rpm}$ for $1.5 \mathrm{~h}$ at $25^{\circ} \mathrm{C}$. The supernatant was removed with a syringe. Since a small amount of DPSL latex particles was still present, we had to separate the PEO from the supernatant in order to determine the unadsorbed PEO concentration. The supernatant was injected into a GPC column. Adsorbance was determined from the concentation change calculated by integrating the peak area of the chromatogram of

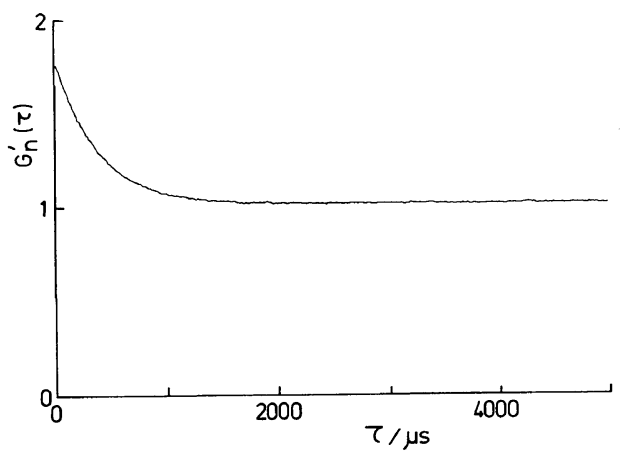

Figure 1. Plot of the normalized photocount autocorrelation function $G_{n}{ }^{\prime}(\tau)$ of DPSL (109 nm diameter) in water at $25^{\circ} \mathrm{C}$ against delay time $\tau$ in $\mu \mathrm{s}$. $C_{\mathrm{L}}=2 \times 10^{-5}$ $\mathrm{g} \mathrm{ml}^{-1} ; \theta=60^{\circ}$; the clock rate, $10 \mu \mathrm{s}$; the channel number, 500 ; and the accumulation number is 1,000 .
PEO, which was found to be separated from the latex peak.

\section{RESULTS AND DISCUSSION}

\section{Estimation of Latex Diameter by QELS}

A plot of the normalized photocount autocorrelation function $G_{n}{ }^{\prime}(\tau)$ against the delay time $\tau$ for DPSL in water at the latex concentration of $C_{\mathrm{L}}=2 \times 10^{-5} \mathrm{~g} \mathrm{ml}^{-1}$ is shown in Figure 1. This curve can be fitted by eq 2 with an appropriate $\beta$ value; this is due to DPSL's being nearly monodisperse in size and shape. The $D q^{2}$ values determined for DPSL at angles from 10 to $150^{\circ}$ are plotted against $q^{2}$ in Figure 2. The translational diffusion coefficient $D$, which should be independent of scattering angle, was estimated from the slope of the straight line. Figure 3 shows that the plots of $D q^{2}$ against $q^{2}$ for the five samples of PSL series are also represented by straight lines over a wide range of scattering angle. Therefore, it is reasonable to as-

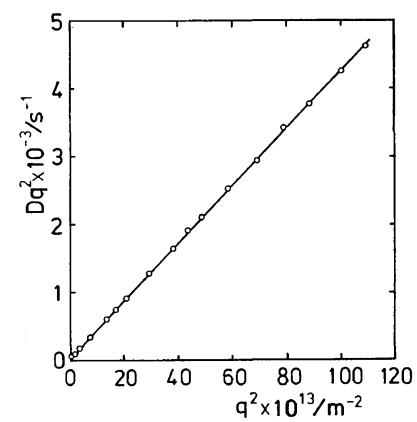

Figure 2. Plot of $D q^{2}$ against $q^{2}$ for DPSL over a scattering angle range from 10 to $150^{\circ}$.

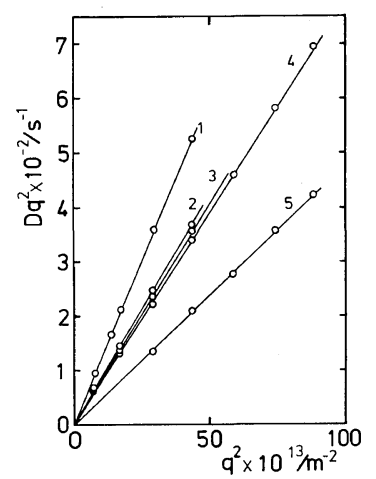

Figure 3. Plot of $D q^{2}$ against $q^{2}$ for PSL series. 1, PSL1; 2, PSL-2; 3, PSL-3; 4, PSL-4; 5, PSL-5. 
Table I. PS latex diameters obtained by QELS and electron microscopy

\begin{tabular}{lccccc}
\hline & & & \multicolumn{2}{c}{ Diameters $/ \mathrm{nm}$} \\
\cline { 4 - 5 } Samples & $D / 10^{-13} \mathrm{~m}^{2} \mathrm{~s}^{-1}$ & $R_{\mathrm{h}} / \mathrm{nm}$ & $\begin{array}{c}\text { from } \\
\text { QELS }\end{array}$ & $\begin{array}{c}\text { from } \\
\text { EM }\end{array}$ \\
& & & & & \\
\hline & & & & 113 & 109 \\
DPSL & 43.15 & 56.5 & & \\
PSL-1 & 12.2 & 200 & 400 & 384 \\
PSL-2 & 8.48 & 288 & 576 & 543 \\
PSL-3 & 8.16 & 299 & 598 & 562 \\
PSL-4 & 7.84 & 311 & 622 & 600 \\
PSL-5 & 4.81 & 507 & 1014 & 979 \\
\hline
\end{tabular}

sume that reliable $D$ values may be estimated from the plot of $G_{n}{ }^{\prime}(\tau)$ against $\tau$ at only one scattering angle, e.g., $45^{\circ}$ or $60^{\circ}$.

The Stokes radii of latex particles $R_{\mathrm{h}}$ were calculated from the measured $D$ values using eq 3 . The values of $D$ and $R_{\mathrm{h}}$ of polystyrene latex particles obtained by QELS are shown in the second and third columns of Table I, respectively. The fifth column gives the particle diameters obtained by EM, which may be compared with the diameters estimated by the QELS method $\left(=2 R_{\mathrm{h}}\right)$. These two sets of diameter values are in good agreement, but the EM values are systematically a few percent smaller than the QELS values. Moreover, it may be concluded that the QELS method is useful for estimating latex diameters ranging from 0.1 to $1.0 \mu \mathrm{m}$.

\section{Adsorption of Poly(oxyethylene) onto DPSL Latex Surfaces}

The molecular characteristics of PEO are shown in Table II. The polydispersity indicies, $M_{w} / M_{n}$, appear to be nearly one, and our PEO samples may be considered almost monodisperse. From the intrinsic viscosity data, the Mark-HouwinkSakurada viscosity equation for PEO in water at $25^{\circ} \mathrm{C}$ was established as follows:

$$
[\eta]=5.94 \times 10^{4} M_{w}^{0.657}
$$

The thickness of the adsorbed layer $t_{\mathrm{h}}$ on DPSL latex particles estimated by QELS is plotted against the adsorption time in Figure 4 for a constant polymer concentration. The values of $t_{\mathrm{h}}$ initially increase with adsorption time and then reach a constant within $2 \mathrm{~h}$. On the other hand, it was found in previous work ${ }^{10}$ that adsorbance slowly approached equilibrium in contrast to thickness; in fact, the equilibrium adsorbance was obtained in 1 day. Thickness was thus determined from $D$ values measured by QELS with the latex PEO mixed solutions left standing for two days. From the plot of $G_{n}{ }^{\prime}(\tau)$ against $\tau$, the DPSL with adsorbed polymer layers also seemed to be monodisperse. For example, the measurement at scattering angle $60^{\circ}$ on the PEO-130-latex mixture of the polymer concentration $6.4 \times 10^{-4} \mathrm{~g} \mathrm{ml}^{-1}$ gave $338 \mathrm{~s}^{-1}$ for $\Gamma_{\mathrm{D}}$ by the single exponential fitting, and $345 \mathrm{~s}^{-1}$ by the cumulant data analysis. Hence, the ratio of the second moment to the square of the first moment in the cumulant series was 0.02 (the ratio is defined by eq 11 in ref 22). No flocculation was observed.

The values of $t_{\mathrm{h}}$ thus obtained are plotted against the bulk polymer concentration $C_{\mathrm{p}}$ in Figure 5 . The latex concentration $C_{\mathrm{L}}$ was kept at $1.1 \times 10^{-5}$ $\mathrm{g} \mathrm{ml}^{-1}$ for all QELS measurements. It was assumed that the $C_{\mathrm{L}}$ was low enough for the concentration dependence of $D$ to be ignored. For all PEO samples, thicknesses at bulk polymer concen-

Table II. Molecular characteristics of poly(oxyethylene) and measured values of equilibrium thickness and equilibrium adsorbance

\begin{tabular}{|c|c|c|c|c|c|c|c|c|}
\hline \multirow{2}{*}{ Samples } & \multicolumn{2}{|c|}{$M_{w} / 10^{4} \mathrm{~g} \mathrm{~mol}^{-1}$} & \multirow{2}{*}{$\frac{[\eta]}{\mathrm{dl} \mathrm{g}^{-1}}$} & \multirow{2}{*}{$\begin{array}{c}M_{w} \\
M_{n}\end{array}$} & \multirow{2}{*}{$\frac{\left\langle S^{2}\right\rangle^{1 / 2}}{\mathrm{~nm}}$} & \multirow{2}{*}{$\frac{t_{\mathrm{h}}}{\mathrm{nm}}$} & \multirow{2}{*}{$\frac{\delta}{\mathrm{mg} \mathrm{m}^{-2}}$} & \multirow{2}{*}{$\begin{array}{c}r_{\mathrm{a}} \\
\mathrm{nm}\end{array}$} \\
\hline & GPC & LS & & & & & & \\
\hline PEO-2 & 2.1 & - & 0.41 & 1.15 & 6.5 & $14 \pm 2$ & $1.8 \pm 0.5$ & 4.4 \\
\hline PEO-4 & 4.6 & - & 0.68 & 1.03 & 10.0 & $20 \pm 2$ & $2.1 \pm 0.5$ & 6.2 \\
\hline PEO-8 & 8.0 & - & 0.97 & 1.03 & 13.5 & $29 \pm 2$ & $3.2 \pm 0.5$ & 7.7 \\
\hline PEO-16 & 16 & 14.3 & 1.47 & 1.04 & 19.1 & $44 \pm 3$ & $3.8 \pm 0.5$ & 11.1 \\
\hline PEO-30 & 32 & 28.4 & 2.45 & 1.06 & 29.4 & $61 \pm 4$ & $4.2 \pm 0.5$ & 16.8 \\
\hline PEO-70 & 73 & 69.0 & 4.13 & 1.10 & 45.5 & $99 \pm 3$ & $4.0 \pm 1.0$ & 30.1 \\
\hline PEO-130 & 130 & 127 & 6.15 & 1.12 & 63.6 & $156 \pm 5$ & $6.0 \pm 0.5$ & 44.6 \\
\hline
\end{tabular}




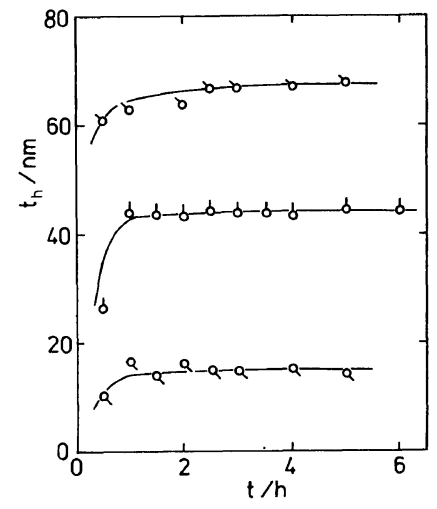

Figure 4. The hydrodynamic thickness $t_{\mathrm{h}}$ of adsorbed layer plotted as a function of adsorption time. Samples: upper, PEO-70 $\left(C=2.25 \times 10^{-6} \mathrm{~g} \mathrm{ml}^{-1}\right)$; middle, PEO$30\left(C=2.33 \times 10^{-4} \mathrm{~g} \mathrm{ml}^{-1}\right)$; lower, PEO-4 $(C=$ $\left.3.99 \times 10^{-4} \mathrm{~g} \mathrm{ml}^{-1}\right)$.

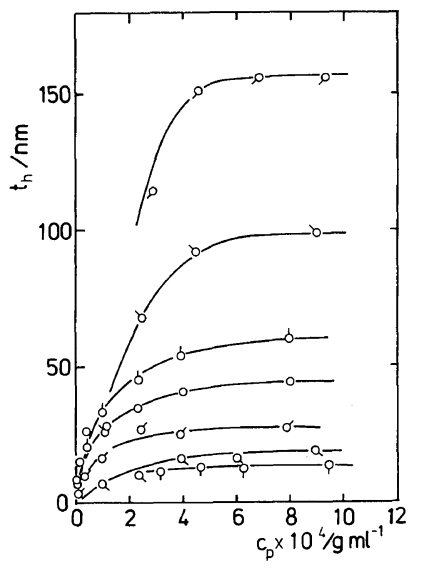

Figure .5. Plot of the adsorbed layer thickness against bulk polymer concentration $C$. Samples: $\bigcirc$, PEO-130; O, PEO-70; $\bigcirc$, PEO-30; O, PEO-16; $\sigma$, PEO-8; $\mathrm{O}$, PEO-4;, , PEO-2.

trations higher than $4 \times 10^{-4} \mathrm{~g} \mathrm{~mol}^{-1}$ were in the plateau region of the $t_{\mathrm{h}}$ against $C_{\mathrm{p}}$ plots. The measured values of equilibrium thickness are shown in the seventh column of. Table II for comparison with the radius of gyration of an isolated polymer in bulk solutions. The radii of gyration were estimated by Flory-Fox equation, ${ }^{23}$

$$
[\eta]=\Phi^{\prime} \frac{\left\langle S^{2}\right\rangle^{3 / 2}}{M}
$$

where $\Phi^{\prime}$ was assumed to be $3.08 \times 10^{22}$.

An example of the adsorption isotherm is shown

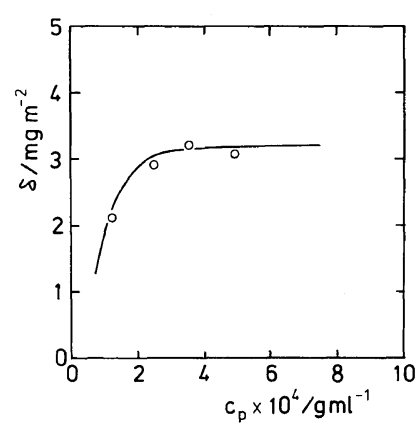

Figure 6. Example of the adsorption isotherms for poly(oxyethylene) on the polystyrene latex DPSL. Sample: PEO-8.

for PEO-8 in Figure 6. The plateau region of the adsorbance was reached at $C_{\mathrm{p}}=4 \times 10^{-4} \mathrm{~g} \mathrm{ml}^{-1}$ Measurement of hydrodynamic thickness required that the plateau regions of both the adsorbance and the thickness be reached in the same $C_{\mathrm{p}}$ region. The values of equilibrium adsorbance, $\delta \mathrm{mg} \mathrm{m}^{-2}$, are shown in Table II.

\section{Conformation of Adsorbed Poly(oxyethylene)}

Hydrodynamic techniques make possible measurement of the hydrodynamic thickness $t_{\mathrm{h}}$ of adsorbed polymer layers, i.e., the distance of the plane of shear from the interface. Experimentally, it has been demonstrated by many authors ${ }^{13-15,24,25}$ that measured $t_{\mathrm{h}}$ are almost twice the radii of gyration of free coils in solution. This fact is also confirmed in this study. Nevertheless, the relationship between $t_{\mathrm{h}}$ and the actual thickness of the adsorbed layer is as yet not clear. On a theoretical basis, assuming that the segment distribution normal to the surface is exponential, which is the case with the loop-train conformation, Varoqui and Dejardin $^{16}$ and de Gennes ${ }^{18}$ concluded that $t_{\mathrm{h}}$ is proportional to but several times larger than the root-mean-square thickness of the loops of adsorbed polymers. On the other hand, Rowland and Eirich $^{24}$ came to the conclusion that $t_{\mathrm{h}}$ is almost identical to the root-mean-square thickness of an adsorbed polymer layer determined by ellipsometry. Thus, at the present stage, the physical meaning of $t_{\mathrm{h}}$ is still obscure. If tails exist, the theoretical calculation of Varoqui and Dejardin ${ }^{16}$ is no longer applicable for estimating adsorbed polymer thickness. Therefore, we assume the hydrodynamic thickness estimated by the QELS method to be an 


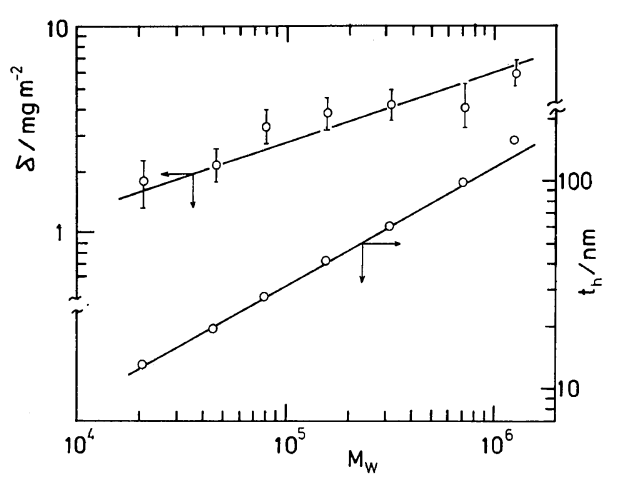

Figure 7. Double logarithmic plots of adsorbed layer thickness $t_{\mathrm{h}}$ against $M_{w}$ of poly(oxyethylene) (lower) and those of adsorbance $\delta$ against $M_{w}$ (upper). The straight lines show apparent molecular weight dependence.

actual adsorbed thickness.

At very low bulk polymer concentrations, both the hydrodynamic thickness and the adsorbance are very small, as shown in Figures 5 and 6. Moreover, the thickness appears to be almost independent of molecular weight. These features are in conformity with theoretical predictions for weak adsorption of polymers from extremely dilute solution, where the adsorbed polymers take on a flattened conformation. With further increase in bulk polymer concentration, thickness increases sharply and comes to depend on molecular weight.

The adsorbance $\delta$ and the hydrodynamic thickness $t_{\mathrm{h}}$ obtained in the plateau region, as summarized in Table II, increase with increasing molecular weight. These values are plotted against molecular weight on a double logarithmic scale in Figure 7. The molecular weight dependence of $\delta$ and $t_{\mathrm{h}}$ was found from the best-fit lines to be $M^{0.34}$ and $M^{0.56}$, respectively.

The fact that the hydrodynamic thickness in the plateau region is nearly twice the radius of gyration of PEO in water leads to the conclusion that the conformation of adsorbed PEO in the plateau should perhaps be a rigid sphere of radius equal to the root-mean-square radius of gyration of a free random coil. ${ }^{15}$ However, the radius $r_{\mathrm{a}}$ per PEO molecule occupied at the interface calculated from $\delta$ is much smaller than $\left\langle S^{2}\right\rangle^{1 / 2}$, as shwon in Table II. Thus, we must assume a considerable overlap or compression of the adsorbed PEO molecules. Since water is a good solvent for PEO, considerable force needed to penetrate or compress the polymer mol- ecules. At the theta point, both theoretically and experimentally, it was found that the thickness of the adsorbed polymer layer is proportional to the square root of the molecular weight. Hence, we assume that the measured $t_{\mathrm{h}}$ increases to a value $\alpha_{\mathrm{t}}$ times larger than $t_{\mathrm{h}}{ }^{0}$ at the theta temperature by the excluded-volume effect or by compression in the adsorbed layer, i.e.,

$$
t_{\mathrm{h}}=k \alpha_{\mathrm{t}} t_{\mathrm{h}}{ }^{0}=k^{\prime} \alpha_{\mathrm{t}} M^{0.5}
$$

where $k$ and $k^{\prime}$ are constants. An attempt to measure the hydrodynamic thickness by QELS under the theta condition, which was realized by the addition of an inorganic salt, was unsuccessful, since the polymer covering latex particles flocculated. However, from the molecular weight dependence of $t_{\mathrm{h}}$, i.e.,

$$
t_{\mathrm{h}} \propto M^{0.56}
$$

we obtain,

$$
\alpha_{t} \propto M^{0.06}
$$

If $\alpha_{t}$ is associated only with the excluded-volumeeffect in the adsorbed layer, we may put,

$$
\alpha_{t}=\alpha_{\eta}=\left([\eta] /[\eta]_{\Theta}\right)^{1 / 3}
$$

since $t_{\mathrm{h}}$ and intrinsic viscosity are hydrodynamic quantities. The following viscosity equation at the theta temperature was estimated from the Stockmayer-Fixman plot, ${ }^{26}$

$$
[\eta]_{\Theta}=2.00 \times 10^{-3} M_{w}^{0.5}
$$

with this relation, the molecular weight dependence of $\alpha_{\eta}$ is derived to be $\alpha_{\eta} \propto M^{0.05}$, since $[\eta] \propto M^{0.657}$ from eq. 4. The molecular weight dependences of $\alpha_{t}$ and $\alpha_{\eta}$ thus obtained are quite consistent with each other.

In conclusion, the adsorbed layer thickness in a good solvent is also equal to twice the radius of gyration of PEO in a bulk solution. The molecular weight dependence of the expansion factor $\alpha_{t}$ may be explained in terms of the hydrodynamic excluded-volume effect.

\section{REFERENCES}

1. A. Silberberg, J. Chem. Phys., 48, 2835 (1968).

2. E. A. DiMarzio, J. Chem. Phys., 42, 2101 (1965).

3. C. A. J. Hoeve, J. Chem. Phys., 44, 1505 (1966); J. Poly. Sci., C, 34, 1 (1971).

4. R. J. Roe, J. Chem. Phys., 44, 4264 (1966).

5. F. Th. Hesselink, J. Phys. Chem., 75, 65 (1971). 
6. F. Th. Hesselink, A. Vrij, and J. Th. G. Overbeek, J. Phys. Chem., 75, 2094 (1971).

7. J. M. H. M. Scheutjens and G. J. Fleer, J. Phys. Chem., 83, 1619 (1979).

8. R. R. Stromberg, D. J. Tutas, and E. Passaglia, $J$. Phys. Chem., 69, 3955 (1965).

9. H. Gebhard and E. Killmann, Angew. Makromol. Chem., 53, 171 (1976).

10. A. Takahashi, M. Kawaguchi, H. Hirota, and T. Kato, Macromolecules, 13, 884 (1980).

11. M. Kawaguchi and A. Takahashi, J. Polym. Sci., Polym. Phys. Ed., 18, 943 (1980).

12. A. Takahashi, M. Kawaguchi, and T. Kato, Polym. Sci. Technol., Ser. B, 12, 729 (1980).

13. M. J. Garvey, Th. F. Tadros, and B. Vincent, $J$. Colloid Interface Sci., 55, 440 (1976).

14. B. W. Morrissey and C. C. Han, J. Colloid Interface Sci., 65, 423 (1978).

15. M. J. Garvey, Th. F. Tadros, and B. Vincent, $J$. Colloid Interface Sci., 49, 57 (1974).

16. R. Varoqui and P. Dejardin, J. Chem. Phys., 66, 4395
(1977).

17. E. Pefferkorn, P. Dejardin, and R. Varoqui, $J$. Colloid Interface Sci., 63, 353 (1978).

18. P. G. De Gennes, J. Physique, 37, 1445 (1976).

19. H. Shariff, H. Sontheimer, and B. Vollmert, Angew, Makromol. Chem., 42, 167 (1975).

20. G. Matsumoto, H. Shimizu, and J. Shimada, Rev. Sci. Instrum., 47, 861 (1976).

21. D. E. Koppel, J. Chem. Phys., 57, 4814 (1972).

22. J. C. Brown, P. N. Pusey, and R. Dietz, J. Chem. Phys., 62, 1136 (1975).

23. P. J. Flory and T. G Fox, J. Am. Chem. Soc., 73, 1904 (1951).

24. F. W. Rowland and F. R. Eirich, J. Polym. Sci., A-1, 4, 2033 and 2041 (1966).

25. Th. van den Boomgaard, T. A. King, Th. F. Tadros, H. Tang and B. Vincent, J. Colloid Interface Sci., 66, 68 (1978).

26. W. H. Stockmayer and M. Fixman, J. Polym. Sci., C, 1, 137 (1963). 